\title{
Neuroprotective Effects of Pregabalin Against Spinal Cord Ischemia-Reperfusion Injury in Rats
} \author{
Aysun GOKCE ${ }^{6}$, Bulent ERDOGAN ${ }^{4}$ \\ ${ }^{1}$ Ufuk University, Faculty of Medicine, Department of Neurosurgery, Ankara, Turkey \\ ${ }^{2}$ Dr. Lutfi Kırdar Kartal Training and Research Hospital, Neurosurgery Clinic, Istanbul, Turkey \\ ${ }^{3}$ Canakkale State Hospital, Neurosurgery Clinic, Canakkale, Turkey \\ ${ }^{4}$ Turgut Ozal University, Faculty of Medicine, Department of Neurosurgery, Ankara, Turkey \\ ${ }^{5}$ Polatli State Hospital, Neurosurgery Clinic, Ankara, Turkey \\ ${ }^{6}$ Diskapi Yıldırım Beyazıt Training and Research Hospital, Pathology Clinic, Ankara, Turkey
}

Burak KAZANCl ${ }^{1}$, Selcuk OZDOGAN² ${ }^{2}$ Ramazan $\mathrm{KAHVECI}^{3}$, Emre Cemal GOKCE${ }^{4}$, Kazim YIGITKANLI ${ }^{5}$,

\section{ABSTRACT}

AIM: To evaluate the effect of pregabalin pre-treatment on spinal cord ischemia-reperfusion (I/R) injury and compare with methylprednisolone (MP).

MATERIAL and METHODS: Thirty-two rats were randomly divided into four groups as follow: Group 1 (sham)(n=8), group 2 (ischemia only)( $\mathrm{n}=8)$, group 3 (30 mg/kg pregabalin)( $\mathrm{n}=8)$, and group 4 (30 mg/kg methylprednisolone)( $\mathrm{n}=8)$. Laparotomy was performed without aortic clamp in the sham group. All animals were sacrificed 24 hours after surgery. The spinal cord tissue samples were harvested and caspase-3 activity, tumor necrosis factor-alpha (TNF-a) and Interleukin-1 Beta (IL-1 $\beta$ ) levels, catalase (CAT) activity, glutathione peroxidase (GPx) activity, superoxide dismutase (SOD) levels malondialdehyde (MDA) levels and nitric oxide (NO) levels were analyzed to investigate the effects of different excitatory and inflammatory pathways in mechanism of I/R injury. Ultrastructural and histopathological examinations were carried out. Neurological recovery was measured by Basso, Beattie, Bresnahan (BBB) test and Inclined Plane Test.

RESULTS: Decresead caspase-3 activity and decreased inflammatory markers like TNF-a, IL-1 $\beta$, and decresaed excitotatory pathways like CAT, GPx, MDA, NO and SOD were observed in both pregabalin pre-treatment and MP treatment groups. Pregabalin pre-treatment produced better ultrastructural results compared to MP treatment, as with histopathological examination. Pregabalin group showed better recovery compared to MP treament group according to BBB scoring system.

CONCLUSION: Pregabalin pre-treatmet and MP treatment both has neuroprotective effect on I/R injury by decreasing caspase dependant apoptosis, and inflammatory and oxidative stress markers. In addition, pregabalin pre-treatment had better clinical effects compared to MP treatment.

KEYWORDS: Pregabalin, Methylprednisolone, Spinal cord injury, Neuroprotection

\section{INTRODUCTION}

$\mathrm{D}$ espite the advances in operative and non-operative techniques to minimise the incidence of neurological complications resulting from the spinal cord ischemia and reperfusion $(\mathrm{I} / \mathrm{R})$ injury, paraplegia remains a devastating complication of thoraco-abdominal aortic surgery. I/R injury of the spinal cord activates complex pathological cascades that leads to neuronal damage via necrosis or apoptosis $(8,14)$. Although pathogenic mechanisms of spinal cord l/R injury are not understood completely, it is now well established that oxidative 
stress resulting from the overproduction of reactive oxygen species with the depletion of endogenous antioxidant defense systems plays a pivotal role in the formation of I/R injury $(7,29)$. These oxygen derived free radicals cause lipid peroxidation of highly vulnerable cell membranes and inflammatory cytokine production that further exacerbate secondary neuronal damage (23).

Pregabalin is an agent recently used for neuropathic pain as in diabetic neuropathy, neuralgia and complex regional pain syndrome (13). Gamma-aminobutyric acid (GABA) is the chemical structure of pregabalin. Pregabalin does not bind to the GABA receptors. It binds at the a2 $\delta$ subunit of the voltage-controlled calcium channels that reduces $\mathrm{Ca}^{+2}$ influx at the presynaptic nerve endings and the release of some neurotransmitters, including glutamate and noradrenalin (12).

In this study, pregabalin is used in spinal cord I/R injury in rats. We aimed to investigate its effectiveness on ischemic injury and compare with methylprednisolone therapy.

\section{MATERIAL and METHODS}

The Ethical Animal Laboratuary Experiment Committee of Ankara Training and Research Hospital approved this study with the file number 136 on August, 09, 2012. The experimental procedures for investigation of the neuroprotective effect of pregabalin against spinal cord ischemia-reperfusion injury in rats. Thirty-two adult Wistar Albino rats were randomly divided into four groups, each group had eight rats weighting $250 \pm 20$ grams. These groups were:

Group 1: Sham group $(n=8)$ - Laparotomy only without aortic clamping. Rats were sacrificed and spinal cord samples were obtained.

Group 2: Ischemia only group $(n=8)$ - Rats underwent transient global spinal cord ischemia. The rats were sacrificed and spinal cord samples were removed 24 hours after ischemia.

Group 3: Methylprednisolone (MP) group $(n=8)$ - IR was performed similar to group 2. Rats received intraperitoneally $30 \mathrm{mg} / \mathrm{kg}$ daily dose of MP (Prednol, Mustafa Nevzat, Turkey) after ischemia.

Group 4: Pregabalin group $(n=8)$ - Rats received intraperitoneally $30 \mathrm{mg} / \mathrm{kg}$ daily dose of pregabalin for one week before IR procedure. Twenty-four hours later, the rats were sacrificed and the spinal cord samples were collected.

\section{Anesthesia and Surgical Procedure}

The conditions of the enviroment that rats kept under were air temperature at $22-25^{\circ} \mathrm{C}$, with appropriate humidity and a 12-hour light cycle and granted free access to food and water. Anesthesia was given with an intraperitoneal injection of $10 \mathrm{mg} / \mathrm{kg}$ xylazine (Rompun, Bayer, Turkey) and 50mg/ kg ketamine (Ketalar, Parke Davis, Turkey), and allowed to breathe spontaneously. A rectal probe was inserted, and the animals were positioned on a heating pad that maintained the body temperature at $37^{\circ} \mathrm{C}$.

Spinal cord I/R was induced using the previously described method with slight modifications. The subjects were operated in the supine position. The aorta was isolated transperitoneally. Five minutes before occlusion $150 \mathrm{lU} / \mathrm{kg}$ heparin was administered intravenously. The aorta was then cross-clamped just inferior to the left renal artery under a surgical microscope using 2 aneurysm clips (Yasargil FE 721; Aesculap, Tuttlingen, Germany). Pulsation of the femoral artery disappeared. The clamps were removed after 30 minutes and return of the aortic pulse was visually verified. Then, the surgical wound was closed in layers with a sterile $6-0$ silk suture. There was no limitation of food and water after 2 hours postoperatively. Rats in the sham group underwent the same procedure, without clamping the aorta. The animals were sacrificed 24 hours after the operation by injection of $200 \mathrm{mg} / \mathrm{kg}$ pentobarbital (Nembutal, Oak Pharmaceuticals, Lake Forest, IL, USA). Spinal cord segments between L2 and S1 were carefully harvested by laminectomy. Then, spinal cord samples cut into three equal parts. Cranial part of spinal cord samples were examined with light microscope, the second sections were examined for electron microscope and the cleared caudal parts were put in $-80^{\circ} \mathrm{C}$ for analyse.

\section{Biochemical Procedures}

Tissue caspase-3 analysis: Caspase- 3 was analysed using the ELISA kit (Uscn Life Science Inc., Wuhan). Sandwich enzyme immunoassay method was used with this kit. Specific antibody was used to coat the microplatekit that is specific to caspase-3. The results are expressed as $\mathrm{U} / \mathrm{g}$-protein.

Tissue tumor necrosis factor-alpha (TNF- $a$ ) analysis: TNF- a activity was analysed using the ELISA kit. Sandwich enzyme immunoassay method was used with this kit. Specific antibody was used to coat the microplatekit that is specific to TNF- $a$. The results are expressed as U/g-protein.

Tissue Interleukin- 1 beta (IL-1 $\beta$ ) analysis: IL-1 $\beta$ was analysed using the ELISA. Sandwich enzyme immunoassay method was used with this kit. Specific antibody was used to coat the microplatekit that is specific to IL-1 $1 \beta$. The results are expressed as $\mathrm{U} / \mathrm{g}$-protein.

Tissue Glutathione Peroxidase (GPx) analysis: GPx was analysed using the ELISA kit. Sandwich enzyme immunoassay method was used with this kit. Specific antibody was used to coat the microplatekit that is specific to GPx. The results are reported as $\mathrm{U} / \mathrm{g}$-protein.

Tissue catalase (CAT) analysis: CAT was analysed using the ELISA. Sandwich enzyme immunoassay method was used with this kit. Specific antibody was used to coat the microplatekit that is specific to CAT.

Tissue malondialdehyde (MDA) analysis: The tissue MDA levels were examined with a method reported by Ohkawa et al. based on the reaction with thiobarbituric acid (TBA) reaction (22).

Tissue nitric oxide (NO) analysis: For analysing tissue NO levels the method of Miranda et al. (20) was used. The results were reported as $\mathrm{nmol} / \mathrm{mg}$ protein.

Tissue superoxide dismutase (SOD) analysis: SOD (EC 1.15.1.1) was proceeced with the method of Sun et al. (27). Superoxide dismutase levels were reported as $\mathrm{U} / \mathrm{mg}$-protein. 


\section{Histopathological Procedures}

The spinal cord tissues were put in paraffin and fixed with $10 \%$ buffered formalin for 24 hours for all groups. Using a microtome, $5 \mu \mathrm{m}$-thick serial sections were stained with hematoxylin-eosin (H\&E). A neuropathologist observed all tissue sections who was blinded to the study design.

For spinal cord tissue samples, a semiquantitative scoring system was used and both histopathological changes and the neuronal degenerative signs were observed. Histopathological changes were scored as; 0: absent; 1: mild; 2: moderate; and 3: common. Specimens were scored based on these four different parameters. The number of normal motor neurons in the anterior horn of the spinal cord was counted in 3 sections for each animal and then averaged.

\section{Ultrastructural examination}

Semi-thin sections of approximately $2 \mu \mathrm{m}$ in thickness and ultrathin sections of approximately $60 \mathrm{~nm}$ thickness were cut with ultramicrotome. The semi-thin sections were stained with methylene blue and examined under light microscope. After this examination, the tissue blocks were stained with uranyl acetate and lead citrate. All the ultra thin sections were examined using a transmission electron microscope. Myelinated axons, evaluated and scored from 0 to 3 as described by Kaptanoglu et al. (16):

\section{0 : Normal myelinated axon}

1: Myelin configuration separation

\section{2: Myelin configuration interruption}

3: Presence of honeycomb in the myelin configuration

\section{Statistical Analysis}

Data analysis was performed by using IBM SPSS Statistics version 17.0 software (IBM Corporation, Armonk, NY, USA). Whether the distributions of continuous variables were determined by using Shapiro Wilk test. For the evaluation of homogeneity, Levene test was used. While, the mean differences among groups were analyzed by One-Way ANOVA, otherwise, Kruskal Wallis test was applied for comparisons of the medians. Post hoc Tukey HSD or Conover's multiple comparison test were used to know which group differ from which others. A p value less than 0.05 was considered statistically significant.

\section{RESULTS}

Tissue caspase-3 activity: In the ischemia group, caspase 3 activity considerably increased in comparison to sham group $(p<0.0001)$. Compared with the trauma group $(p<0.0001)$, caspase 3 activity considerably decreased both in MP and Pregabalin treatment and there was not any important differences between them $(p=0.9499)$ (Figure 1A).

Tissue TNF-a levels: In the ischemia group, the tissue level of TNF was considerably increased in comparison to sham group $(p<0.0001)$ (Figure $1 B)$. Compared with the trauma group $(p<0.0001)$, tissue levels of TNF were considerably decreased both in MP and Pregabalin treatment and there was not any important differences between them $(p=0.9162)$.

Tissue IL-1 $\boldsymbol{\beta}$ Activity: In the ischemia group, IL-1 $\beta$ activity considerably increased in comparison to sham group $(p<0.0001)$. Compared with the ischemia group $(p<0.0001)$, IL-1 $\beta$ activity was considerably decreased both in MP and Pregabalin treatment and there was not any important differences between them $(p=0.9751)$ (Figure 1C).

Tissue GPx activity: In the ischemia group, tissue GPx activity considerably increased in comparison to sham group $(p<0.0001)$. Compared with the ischemia group $(p<$ 0.0001 ), GPx activity was considerably decreased both in MP and Pregabalin treatment and there was not any important differences between them $(p=0.7750)$ (Figure 1D).

Tissue Catalase Activity: In the ischemia group, catalase activity considerably increased in comparison to sham group $(p<0.0001)$. Compared with the ischemia group, catalase activity was considerably decreased both in MP $(p<0.0001)$ and Pregabalin $(p=0.0002)$ treatment and there was not any important differences between them $(p=0.9803)$ (Figure 1E).

Tissue MDA levels: In the ischemia group, the tissue MDA levels considerably increased in comparison to sham group $(p<0.0001)$. Compared with the ischemia group $(p<0.0001)$, tissue MDA levels were considerably decreased both in MP and Pregabalin $(p=0.7396)$ treatment and there was not any important differences between them $(p=0.7396)$ (Figure 1F).

Tissue NO levels: In the ischemia group, the tissue NO levels considerably increased in comparison to sham group $(p<0.0001)$. Compared with the ischemia group $(p<0.0001)$, tissue NO levels were considerably decreased both in MP and Pregabalin treatment and there was not any important differences between them $(p=0.5088)$ (Figure 1G).

Tissue SOD activity: In the ischemia group, the tissue SOD activity considerably increased in comparison to sham group $(p<0.0001)$. Compared with the ischemia group, tissue SOD activity was considerably decreased both in MP and Pregabalin $(p=0.0031)$ treatment and there was not any important differences between them $(p=0.7361)$ (Figure 1H).

\section{Histopathology Light Microscopy Examination}

The neurons of spinal cord samples from the sham group were not significantly different from normal neurons when they were examined under the light microscope (Figure 2A). In the ischemia group, histological transformations were observed. In this group, congestion and diffuse haemorrhage areas, neural pyknosis, cytoplasmic eosinophilia and loss of cytoplasmic features were seen (Figure 2B). This pathological finding seen in the trauma group was lessened both in MP (Figure 2C), and Pregabalin treatment groups (Figure 2D).

\section{Histopathological Grading}

The ischemia group had considerably higher pathological score compared with the sham group $(\mathrm{p}<0.0001)$. MP and Pregabalin treatment groups both had considerably lower scores than the ischemia group $(p<0.0001)$. There was not any remarkable difference between the MP and the Pregabalin treatment groups $(p=0.8665)$ (Figure $3 A)$. 
(A)

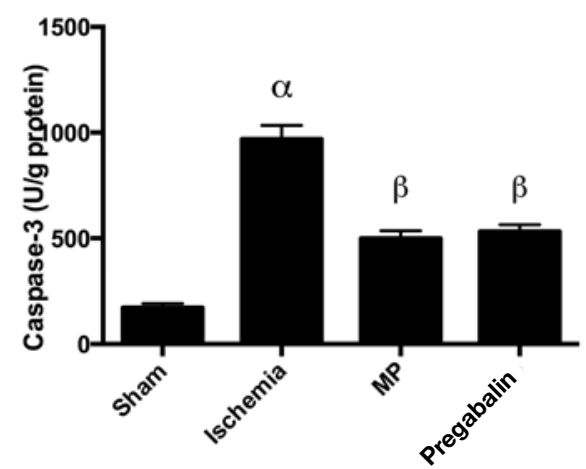

(C)

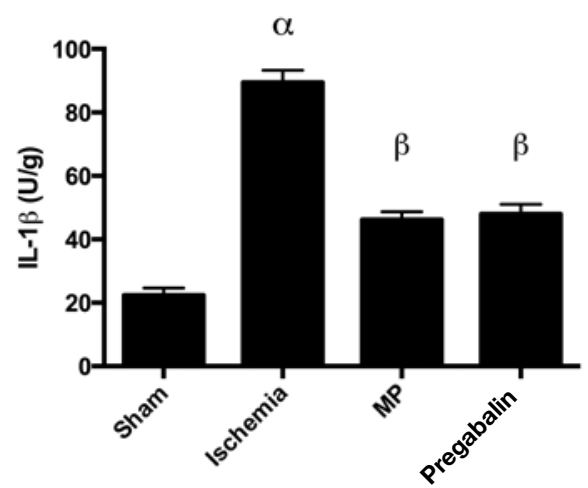

(E)

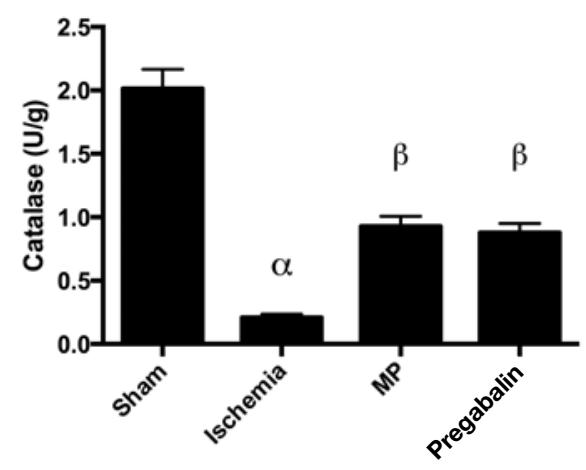

(G)

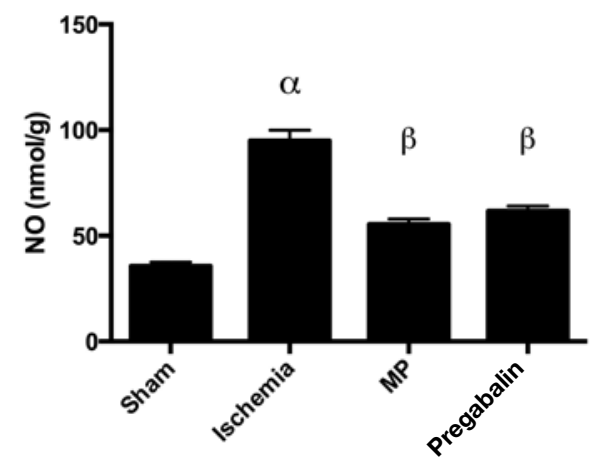

(B)

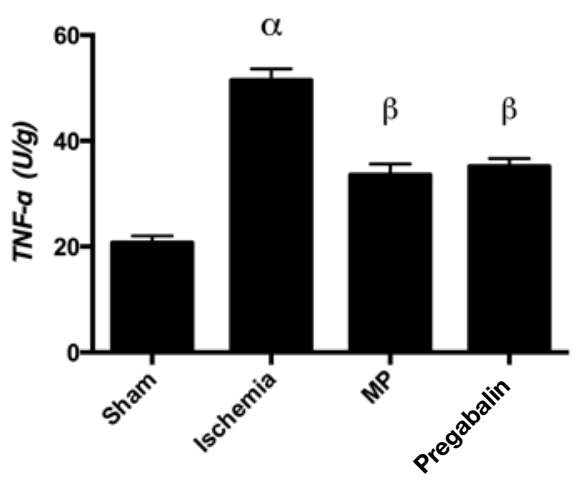

(D)

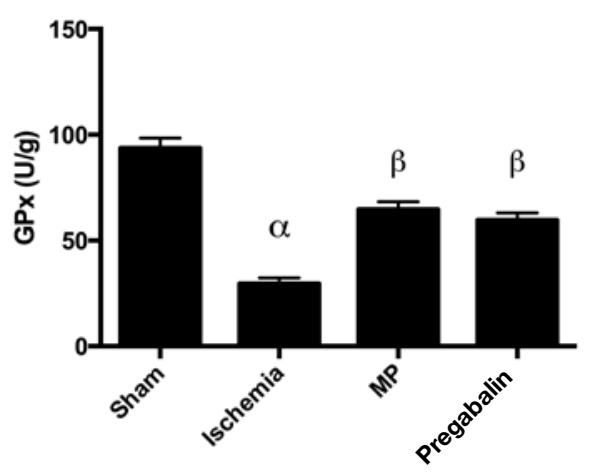

(F)

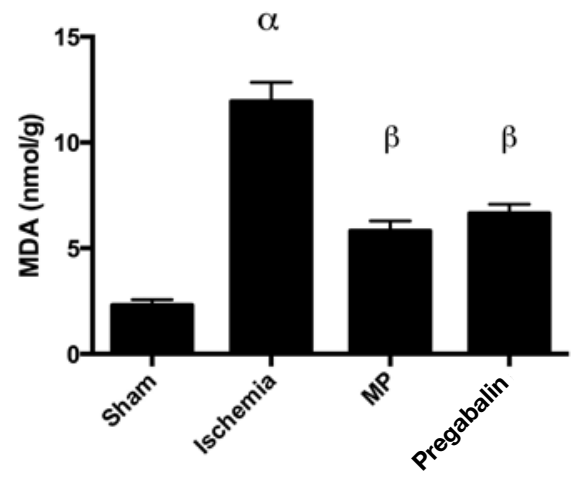

(H)

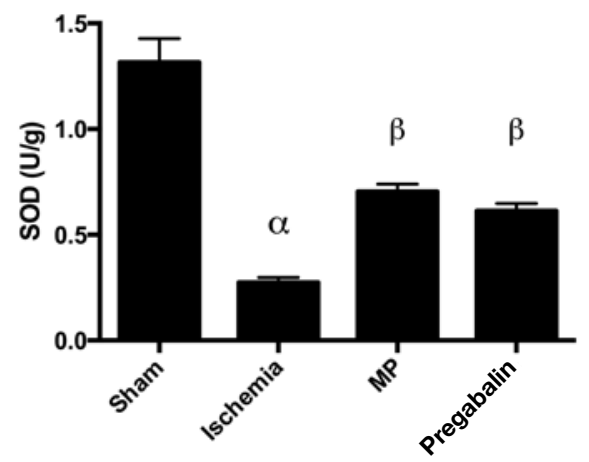

Figure 1A-H: Bar Graphs representing the biochemical pathway results as mean \pm SE. Statistical analysis were done as One-Way ANOVA (Post hoc Tukey comparisons); (alfa) Sham vs Ischemia ( $<<0.0001)$, ( $(B)$ Ischemia vs MP $(p<0.0001)$, (B) Ischemia vs Pregabalin $(p<0.0001)$. There was no statistically significant difference between MP and Pregabalin $(p>0.05)$. 
There was not any remarkable difference between the MP and the Pregabalin treatment groups $(p=0.8665)$ (Figure $3 A)$.

Normal motor neuron numbers in anterior horn of the spinal cord were also evaluated. The number of motor neurons were considerably lower in the ischemia group compared with the sham group $(p<0.0001)$. The number of normal motor neurons in the MP treatment and Pregabalin treatment groups were considerably higher than the ischemia group $(p<0.0001)$. When we consider number of normal motor neurons there is not any statistically considerable difference $(p=0.8329)$ between the MP and Pregabalin treatment groups (Figure 3B).

\section{Electron Microscopy}

There was no ultrastructural examination of the gray matter. All sized myelinated axons were ultrastructurally normal in the white matter (Figure 4A).

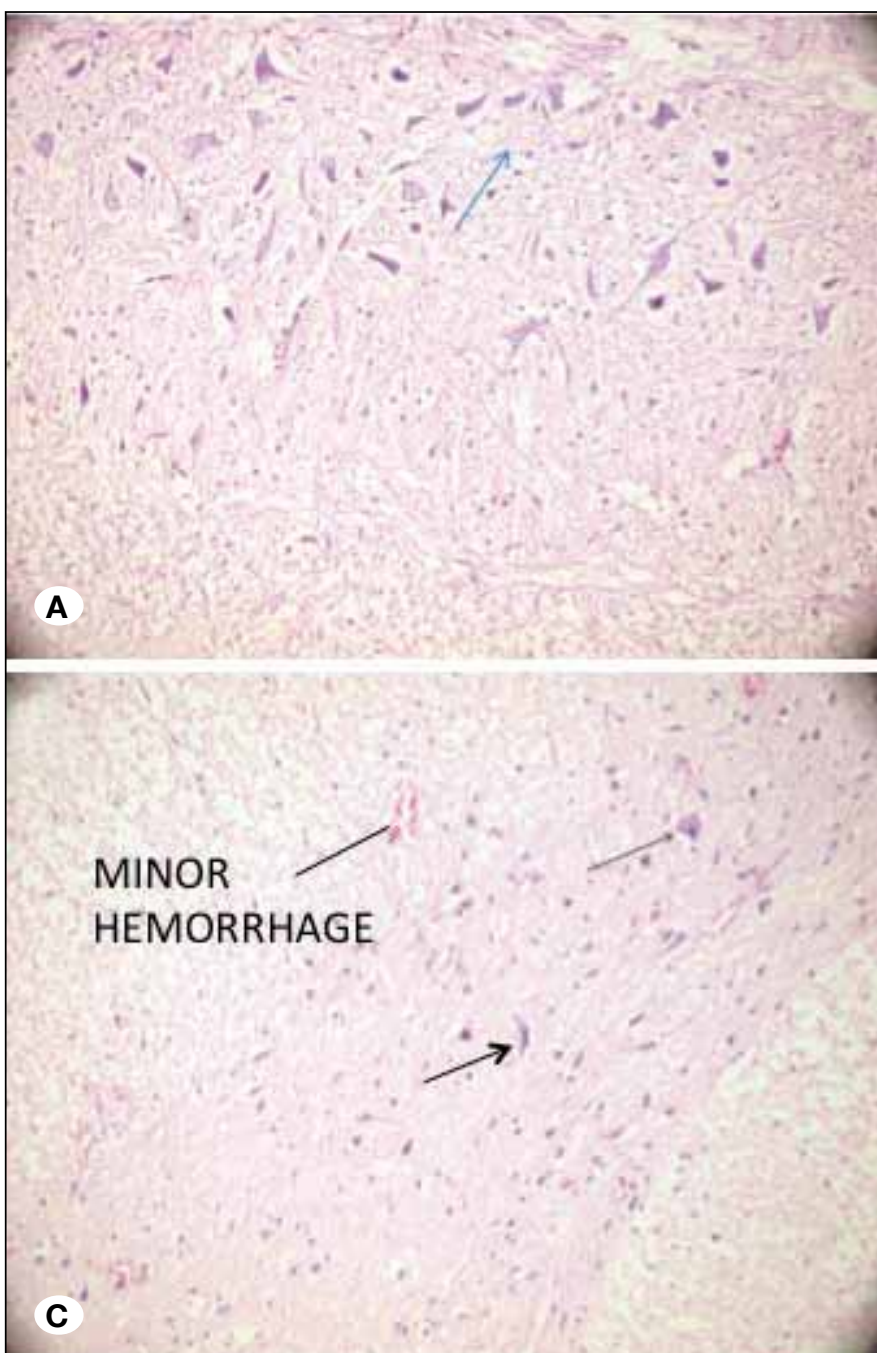

Myelin configuration seperations and also interruptions were observed in all sized, myelinated axons in only ischemia group. Vacuoles and swollen mitocondria were present inside the cytoplasm of neurons in the gray matter. Perineural edema was also present (Figure 4B).

In MP treatment group swollen mitocondria and vacuoles were observed inside the cytoplasm of neurons in the gray matter. Additionally; perineural edema was also present. Separations in myelin configuration were found in small, medium sized large sized myelinated axons in the white matter (Figure 5A).

In Pregabalin pre-treatment group separations and interruptions in myelin configuration were observed in small sized, medium sized and large sized myelinated axons (Figure 5B).

In the ultrastructural examination of the gray matter; no ultrastructural pathology was detected. All of the neurons were ultrastructurally normal (Figure 5C).

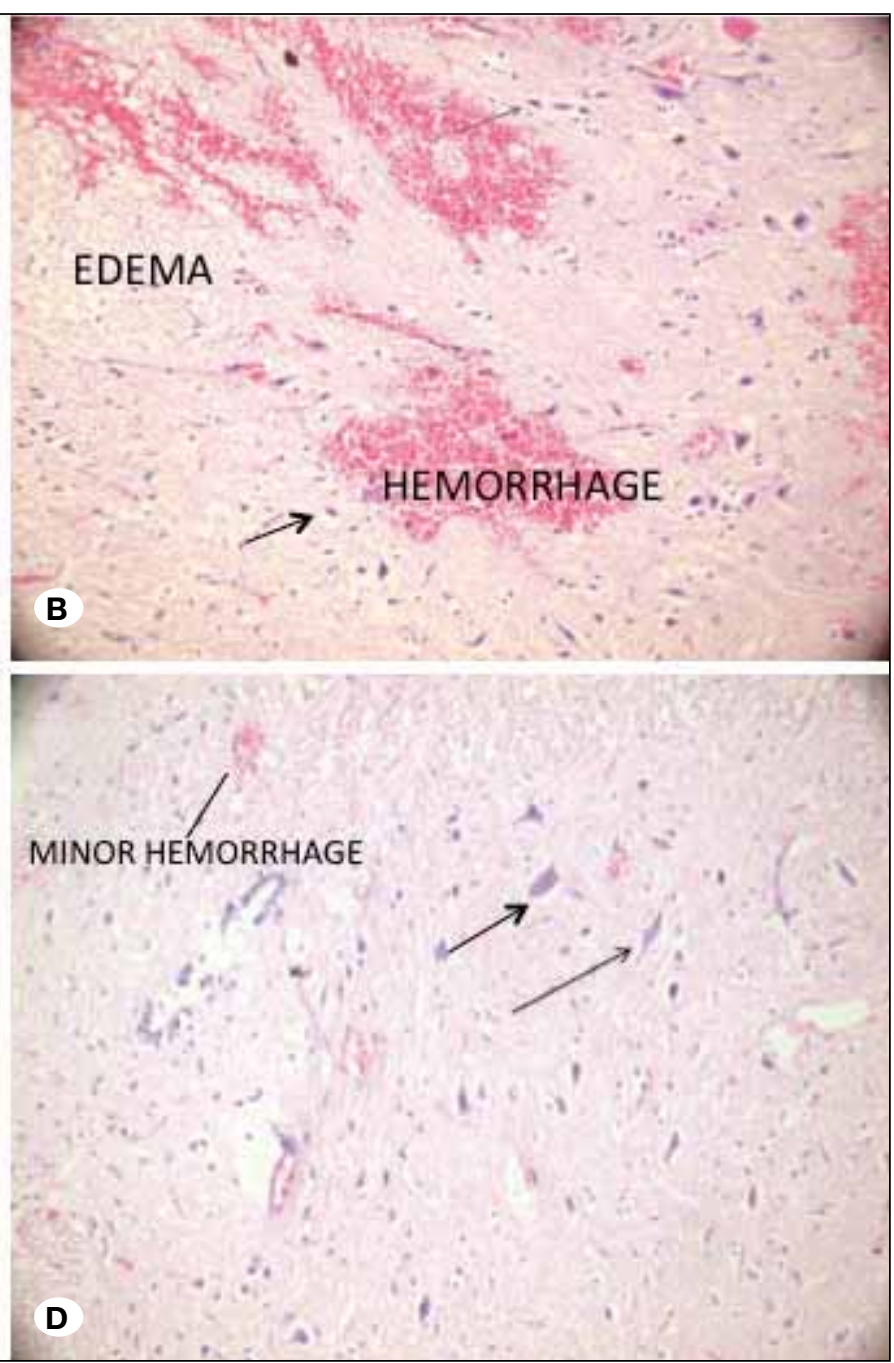

Figure 2: Photomicrographs of 5-mm-thick spinal cord tissue sections from four different groups (H\&E, $\mathrm{x} 40)$. A) Control group revealing spinal cord parenchyma with normal neurons (arrow). B) Ischemia group, showing haemorrhagic areas in spinal cord parenchyma with highly degenerated neurons (arrows). C) MP treatment group, showing less hemorrhagic areas with normal appearing neurons (arrow). (D) Pregabalin treatment group, revealing normal appearing neurons (arrow) with minimal haemorrhagic spinal cord parenchyma (MP: methylprednisolone). 
When we compare ischemic group with sham group the ischemia group showed more disruption in all sized myelinated $(p<0.001)$. MP treatment protected the axons of only small and large-sized myelinated axons from the ischemia $(p=0.0424$ and $p=0.0448$, respectively). Pregabalin treatament could not save any of those myelinated axons (Figure 6A-C).

\section{Neurological Outcomes}

Basso-Beattie-Bresnahan (BBB) test Score: Initial BBB score of rats was 21 (2). Following ischemia, there was a statistically significantly decrease of the mean BBB score in ischemia group was decreased compared with the sham group $(p<0.0001)$. Both the MP and the Pregabalin treatment groups showed better BBB score outcomes compared with the ischemia group $(p<0.0001)$. Additionally Pregabalin pre-treatment group showed better outcome compared to MP treatment group $(p=0.0368)$ (Figure 7A).

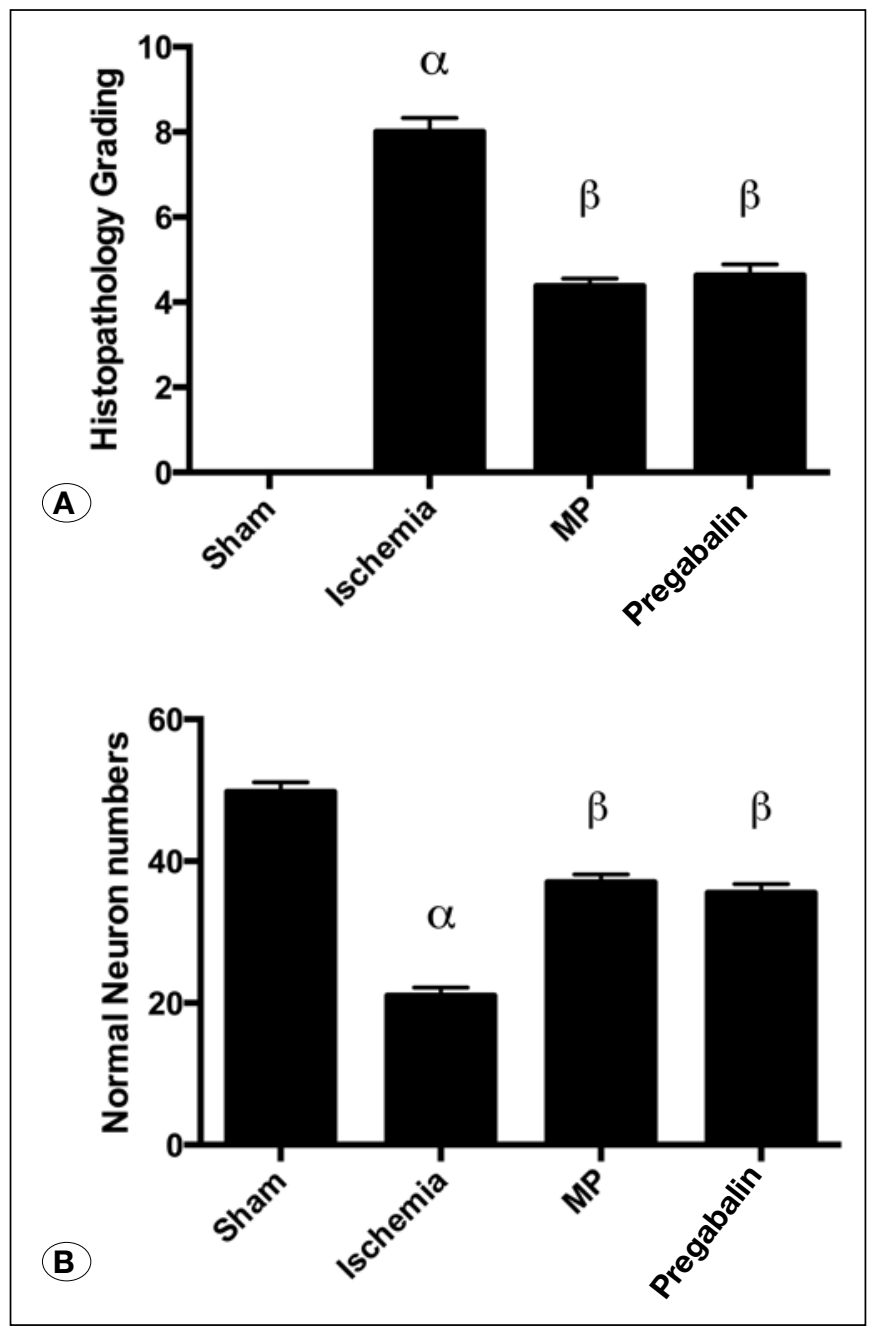

Figure 3A, B: Bar graphs representing the pathology scores of the study groups as mean \pm SE. (alfa) Sham vs Ischemia $(p<0.0001)$, (B) Ischemia vs MP $(p<0.0001)$, (B) Ischemia vs Pregabalin $(p<0.0001)$. There was no statistically significant difference between MP and Pregabalin ( $p>0.05$ ) (Statistical analysis were done as One-Way ANOVA (Post hoc Tukey comparisons).
Inclined Plane Test: The mean angle recorded in the inclined-plane test was significantly lower in the rats in ischemia group compared to sham group $(p<0.0001)$. The rats in MP and Pregabalin treatment groups scored better angles in the inclined-plane test compared to the ischemia group $(p<0.0001$ for both). There was no significant difference between the MP and Pregabalin treatment groups $(p=0.1839)$ (Figure 7B).

\section{DISCUSSION}

Spinal cord I/R injury is a potentially devastating and unpredictable complication of thoraco-abdominal aortic surgery that may result in paraplegia in up to $40 \%$ of patients. Several protective strategies have been attempted to prevent this catastrophic complication, nevertheless an effective therapeutic management has not yet been developed. Although the exact pathophysiological mechanisms of spinal cord $\mathrm{I} / \mathrm{R}$ injury are not still completely understood, it is evidenced that initial ischemic injury, which is paradoxically aggravated by reperfu-
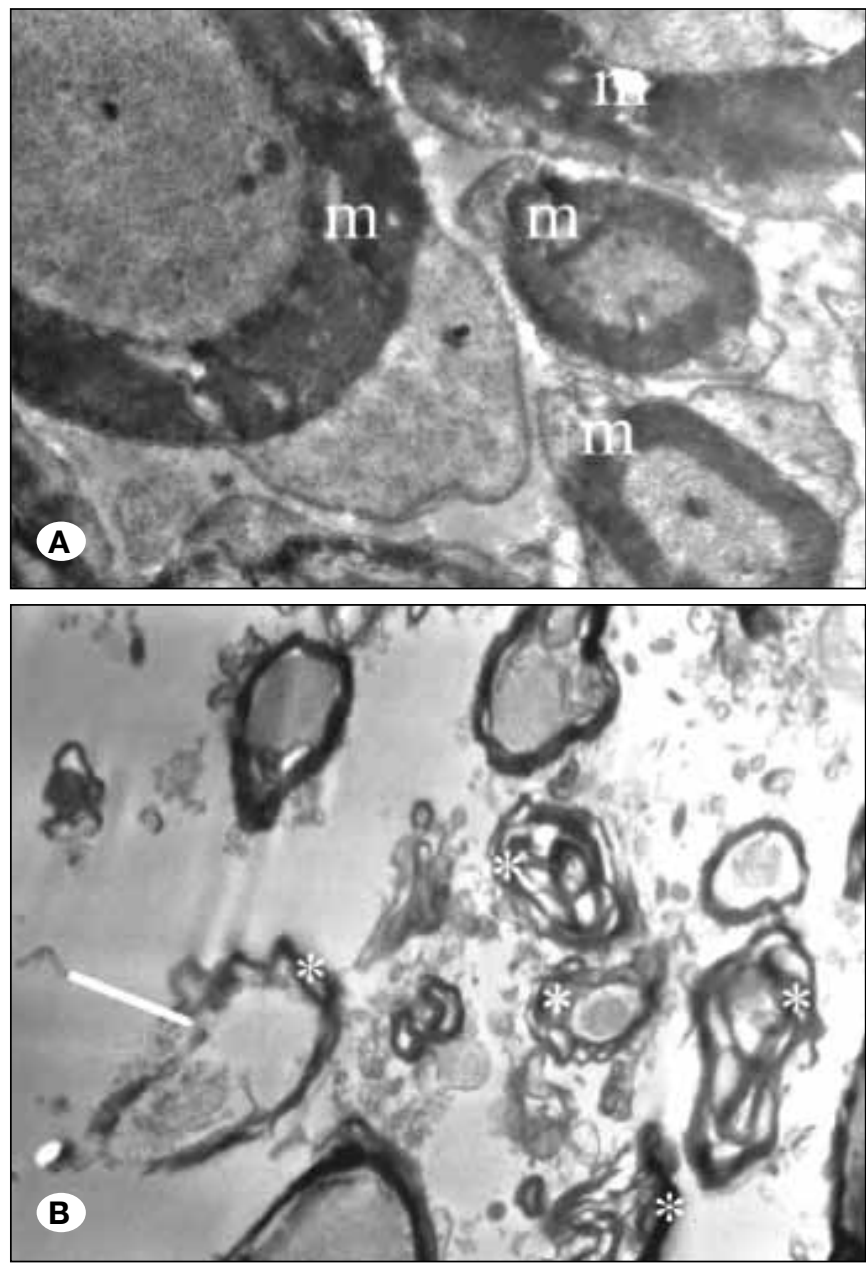

Figure 4: Transmission electron microscopy of the sham and ischemia groups. A) Micrograph shows ultrastructurally normal myelinated axon; B) ischemia reperfusion injury electron micrograph showing seperations (*) and interruptions (arrow) in myelin configuration of myelinated axons. 

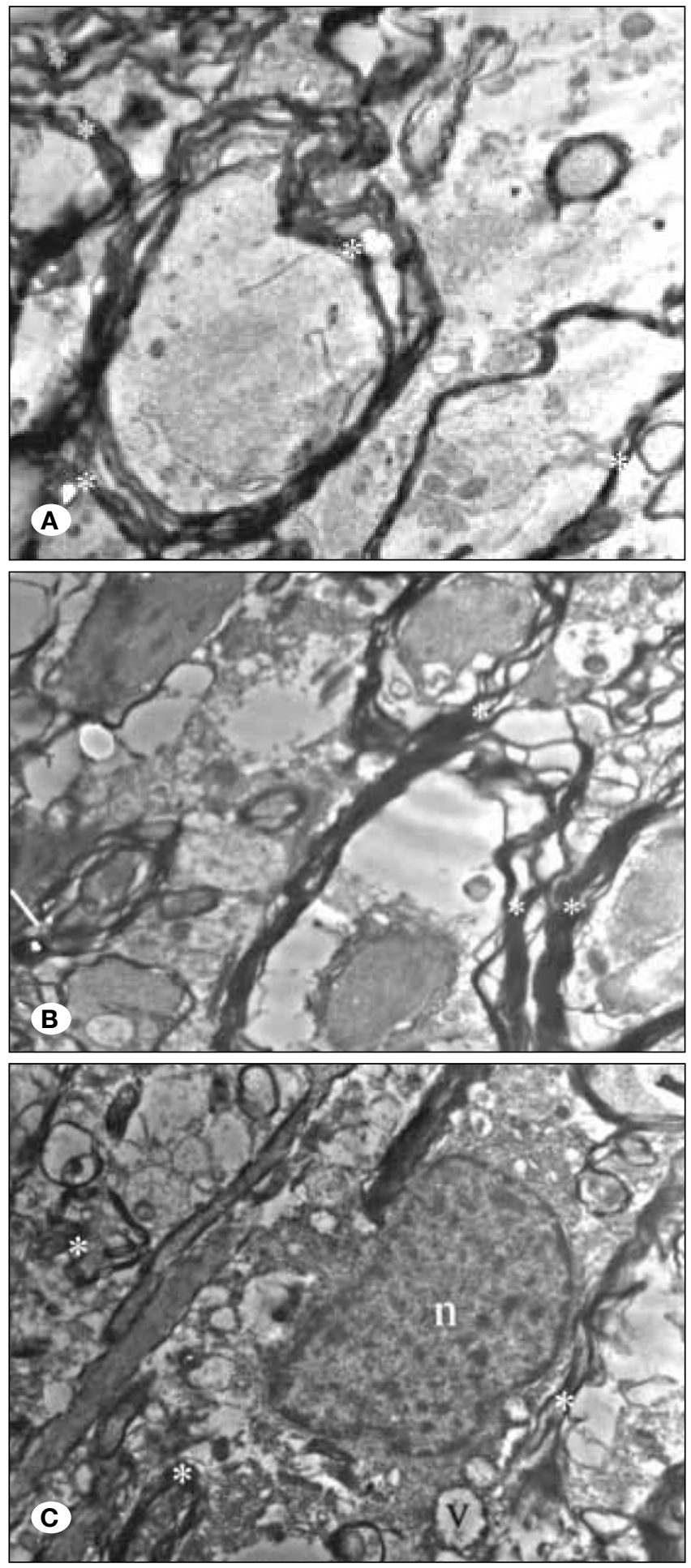

Figure 5: Transmission electron microscopy of the treatment groups. A) Electron micrograph showing seperations in myelin configuration (*) in medium and small sized myelinated axons in MP treatment group. B) Electron micrograph showing seperations $\left(^{*}\right)$ and interruptions (arrow) in myelin configuration of myelinated axons in Pregabalin pre-treatment group.C) Electron micrograph showing a vacuole $(\mathrm{v})$ inside the cytoplasm of a neuron and myelinated axons with seperation in myelin configuration $\left({ }^{\star}\right)$. (n: nucleus of neuron in Pregabalin group).

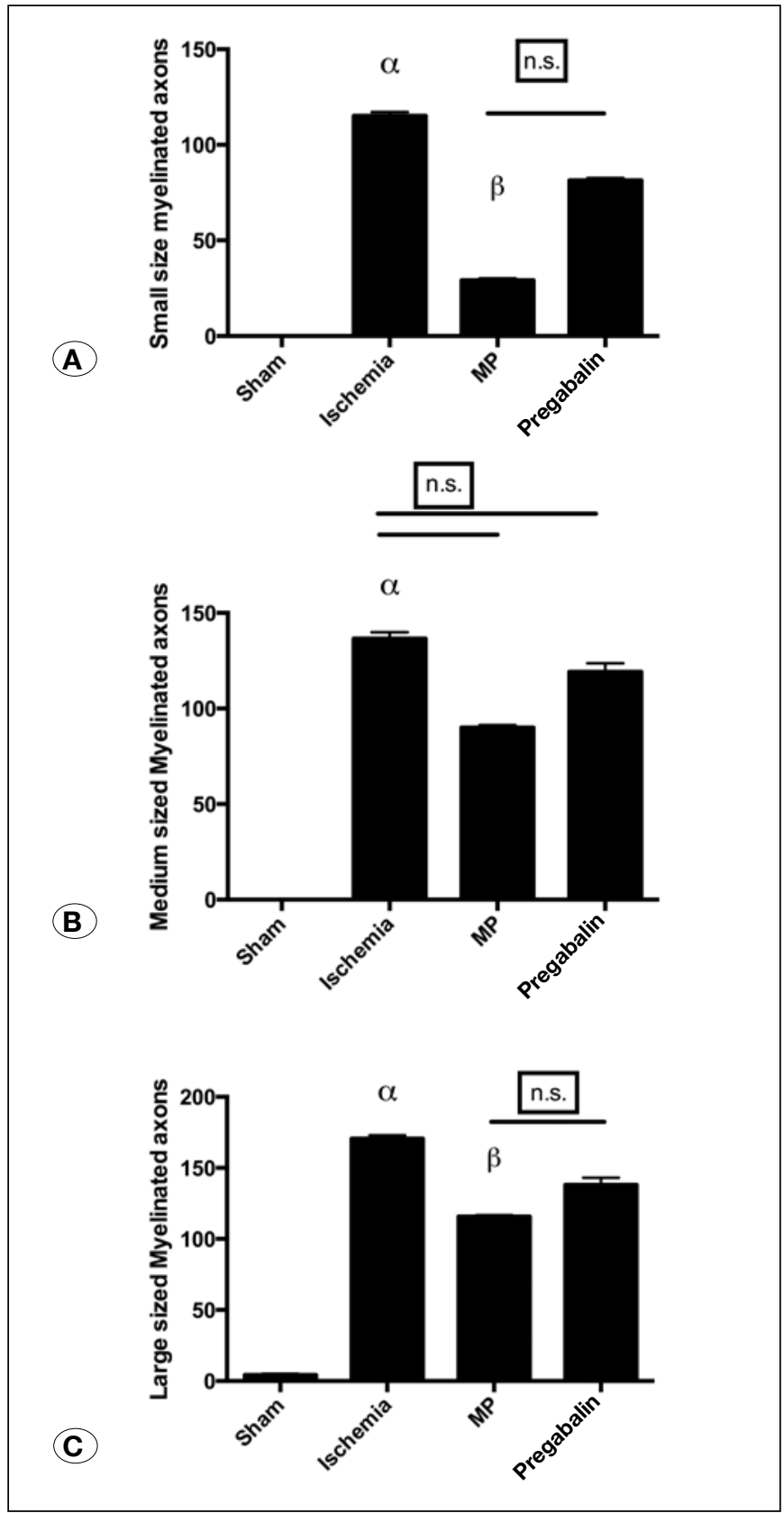

Figure 6: The electron microscopy results showing number of disrupted small-, medium-, and large-sized myelinated axons as mean \pm SE. A) Small sized myelinated axon disruption numbers (alfa) Sham vs Ischemia ( $p<0.001)$, (B) Ischemia vs MP $(p<0.05)$, Pregabalin treatment group was not significantly different from ischemia group and/ (n.s.) not significant difference between MP and Pregabalin treatment groups. B) Medium sized axon disruption numbers (alfa) Sham vs Ischemia $(p<0.001)$, (n.s.) not statistically signicant difference of MP and Pregabalin treatment groups compared to ischemia. ( $p>0.05)$. C) Large sized myelinated axon disruption numbers. (alfa) Sham vs Ischemia $(p<0.001)$, (B) Ischemia vs MP $(p<0.05)$, Pregabalin treatment group was not significantly different from ischemia group and (n.s.) not significant diffrerence between MP and Pregabalin treatment groups. (Statistical analysis was done as Kruskal Wallis, Post hoc Dunn's comparisons). 
sion, causes neuronal tissue damage by activation of several interconnected pathological cascades including glutamatemediated excitotoxicity, oxidative stress, lipid peroxidation, inflammation and apoptosis $(5,15,18)$.

The efficiency and safety of MP treatment has been intensely questioned and leading to discontinuation of its use in some clinical guidelines (6). According to recent studies, MP regimen can not be recommended as a guideline or standard of the acute spinal cord injury treatment (11). However, it still remains as a treatment option because of its role in inhibition of lipid peroxidation and anti-inflammatory and anti-apoptotic effects until it is supplanted by future evidence based therapies (22). As MP is widely used in the experimental models of spinal cord injury, we compared the results of pregabalin with MP.

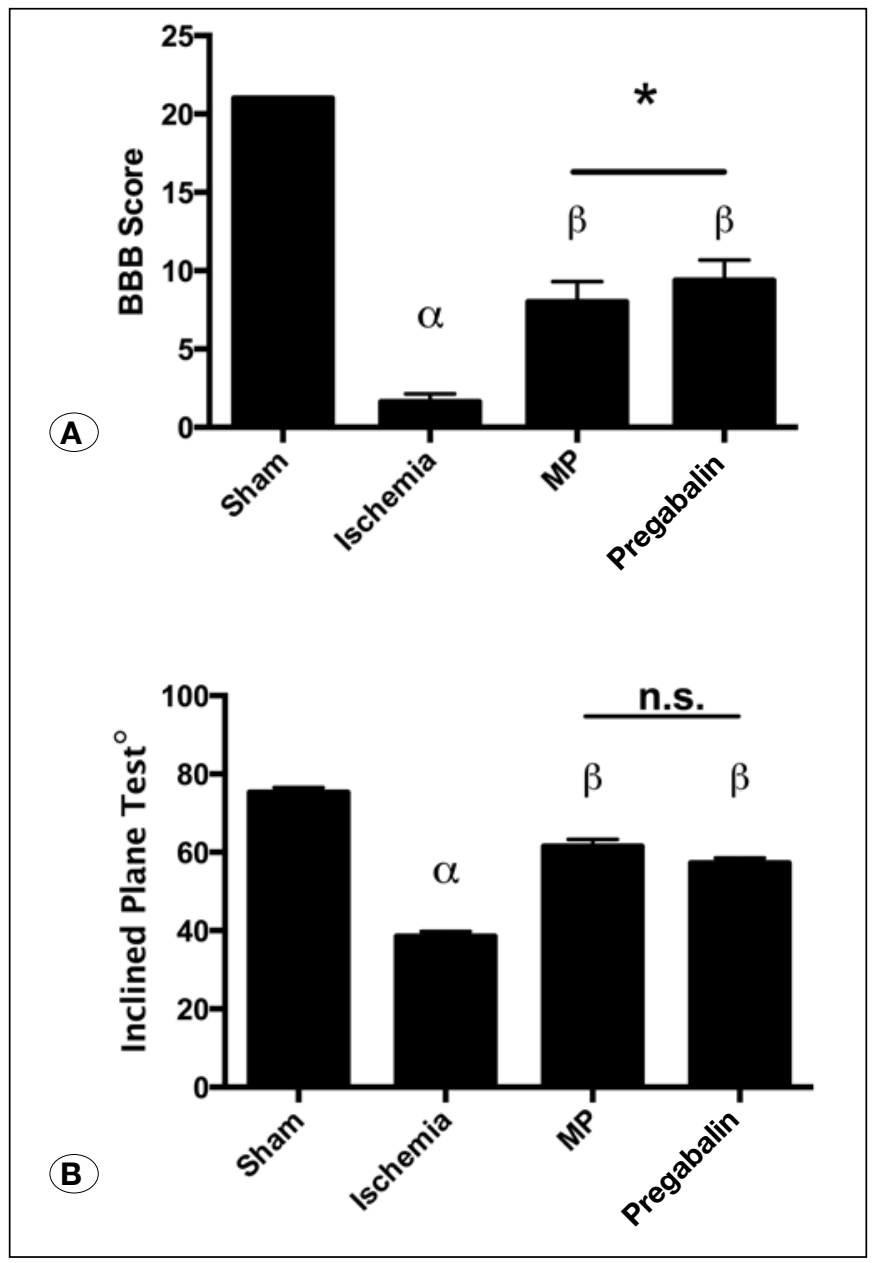

Figure 7: Neurological examination results of the study groups. A) Bar graphs representing BBB (Basso, Beattie, and Bresnahan) scores. (alfa) Sham vs Ischemia $(p<0.0001),(B)$ Ischemia vs MP $(p<0.0001)$, (B) Ischemia vs Pregabalin $(p<0.0001)$. $\left(^{*}\right)$ Pregabalin treatment group showed significantly better scores compared to MP treatment $(p<0.05)$. B) Bar graphs representing angles performed by rats on Inclined Plane Test. (alfa) Sham vs Ischemia $(p<0.0001)$, ( $(B)$ Ischemia vs MP $(p<0.0001)$, ( $($ ) Ischemia vs Pregabalin $(p<0.0001)$. (n.s.) Pregabalin and MP treatment groups were not significantly different $(p>0.05)$. (Statistical analysis was done as One-Way ANOVA, Post hoc Tukey comparisons).
I/R injury was associated with increased oxidative stres (29). Oxidative stress can be defined as an imbalance between the increased production of reactive oxygen species (ROS) and inadequacy of the endogenous antioxidant defense systems (26). Although ROS are frequently generated spontaneously in the living cell during normal metabolism, excessive amount of ROS can damage cellular components including proteins, lipids, and nucleic acids, causing cellular dysfunction (3). Accumulation of ROS cause lipid peroxidation within the cell membrane, resulting in a large amount of malondialdehyde production. Antioxidant enzymes, such as SOD, CAT and GPX, are considered to be a primary defense against ROS-induced cellular damage (30). However, their activities are diminished under highly elevated oxidative stress conditions because of molecular damage. Previous studies showed that pregabalin protect tissues against oxidative stress through increasing in the activities of SOD, GPx and CAT and decreasing the MDA levels in a dose dependent manner $(12,13)$. Consistent with previous results, our study demonstrated that I/R injury causes depletion of endogenous antioxidant enzyme activities and an increase in MDA levels in spinal cord tissue and these pathological changes were also substantially reversed by administration of either pregabalin or MP.

Inflammation is an important pathological process in ischemia, particularly during the acute phase (28). Beside their direct damage to the cell membranes, ROS trigger the infiltration of neutrophils into the injured site of the spinal cord. Neutrophils mediate the inflammatory response of the spinal cord to ischemic injury by producing inflammatory cytokines, such as TNF- $a$ and IL-1B that may recruit other inflammatory cells and contribute to the expansion of ischemic damage after reperfusion through generation of free radicals, such as NO (10). As with other free radicals, NO can lead to exacerbation of lipid, protein, and nucleic acid damage in neurons (9). Thus, the assay of cytokines and nitric oxide in the tissue are useful quantitative indicators for the extent and types of spinal cord I/R injury (20). In the present investigation both Pregabalin pretreated and MP treated gropus showed lower expression of inflammatory markers.

Following spinal cord I/R injury, neuronal cells may be death either by necrosis or apoptosis due to severity of initial insult (1). Neurons that exposed to more severe ischemic insult may be death preferentially by severe membrane failure and necrosis, whereas less severe insults may be more likely to leave neuronal cells injured badly enough to trigger apoptosis, which leads to delayed cell death (17). Apoptotic death of neural cells, especially neurons and oligodendrocytes, disrupts and destroys the axon-myelin structural unit resulting in impaired impulse conduction (4). Cells that undergo apoptosis are autodigested by cysteine proteases known as caspases (19). Caspase-3 is an interleukin converting enzyme and has been implicated as the principal effector of apoptosis in mammalian cells (25). Previous studies demonstrated that caspase- 3 is a reliable marker of apoptosis in spinal cord I/R injury (21). In our study, caspase-3 activity increased significantly as an indicator of caspase dependant apoptosis, which is consistent with previous observations. Previous studies has shown that this phenomenon peaks at $48 \mathrm{hrs}$ and 7 days (13). Excitototoxicity 
is known to be one of the major mechanism to induce apoptosis (24). Overexpressed excitotoxic neurotransmitters like glutamate can induce apoptosis by direct damage to mitochondria (26). The reduced expression of caspase-3 in the pregabalin treatment group may be explained by reduced extracellular glutamate concentration by the pharmacological action of this drug leading to decreased excitotoxicity $(24,25)$. In our study both Pregabalin pre-tretament and MP treatment groups showed decreased caspase-3 activity.

Histopathological examination was performed to support the biochemical results that obtained from the present study. Diffuse pericellular edema and inflammatory cell infiltration, in addition to vascular congestion with neuronal and axonal injury findings, including shrinkage of the cell body, pyknosis of the nucleus, loss of cytoplasmic features and clusters of retraction were observed in both white and gray matter of the spinal cord samples 24 hours after the I/R injury. Also the number of normal motor neurons was significantly decreased. All of these pathological changes were either prevented or alleviated by both pregabalin and MP treatments. Furthermore, pregabalin produced better histopathological results than MP.

In order to gain more detailed data, we also evaluated the ultrastructural changes with a transmission electron microscope (TEM). TEM results revealed that I/R caused significant disruption in small, medium and large-sized myelinated axons. Both the pregabalin and MP treatments protected the small-, medium- and large-sized myelinated axons from I/R injury. Pregabalin also produced better ultrastructural results than MP, as with histopathological examination.

However, this study has some limitations. It was designed as a preliminary observation, and it did not contain neither functional outcomes nor biochemical and histopathological changes occurring in long-run due to high mortality risk of the experimental animals. Additionally, the number of rats in each group may be augmented for applying of these results in clinical use, and the neuroprotective effects of pregabalin may be investigated in a time and dose dependent manner. Although, we could not find a significant difference between pregabalin and MP for each of the biochemical parameters, pregabalin produced better histopathological and ultrastructural results.

\section{CONCLUSION}

Pregabalin and MP protects spinal cord against I/R injury by attenuating lipid peroxidation, inflammatory markers and caspase dependent apoptotic pathways. It also helps to preserve histopathological, ultrastructural findings and helps to improve clinical outcome. Overall pregabalin pretreatment had better clinical outcome compared to MP treatment without any diffrence in biochemical pathways. Further studies may be needed for the establishment of neuroprotective activity of pregabalin in a time- and dose-dependent manner.

\section{ACKNOWLEDGMENT}

We are grateful to Dr. Nurkan Aksoy, Dr. Üçler Kısa and Dr. Mustafa Fevzi Sargon for their contribution to this study.

\section{REFERENCES}

1. Balentine JD: Pathology of experimental spinal cord trauma. I. The necrotic lesion as a function of vascular injury. Lab Invest 39: 236-253, 1978

2. Basso DM, Beattie MS, Bresnahan JC: A sensitive and reliable locomotor rating scale for open field testing in rats. $J$ Neurotrauma 12: 1-21, 1995

3. Beattie MS, Hermann GE, Rogers RC, Bresnahan JC: Cell death in models of spinal cord injury. Prog Brain Res 137: 37-47, 2002

4. Borgens RB, Blight AR, Murphy DJ: Axonal regeneration in spinal cord injury: A perspective and new technique. J Comp Neurol 250: 157-167, 1986

5. Casha S, Yu WR, Fehlings MG: Oligodendroglial apoptosis occurs along degenerating axons and is associated with FAS and p75 expression following spinal cord injury in the rat. Neuroscience 103: 203-218, 2001

6. Chen HC, Fong TH, Lee AW, Chiu WT: Autophagy is activated in injured neurons and inhibited by methylprednisolone after experimental spinal cord injury. Spine 37: 470-475, 2012

7. Cooney SJ, Zhao Y, Byrnes KR: Characterization of the expression and inflammatory activity of NADPH oxidase after spinal cord injury. Free Radic Res 48: 929-939, 2014

8. Crowe MJ, Bresnahan JC, Shuman SL, Masters JN, Beattie MS: Apoptosis and delayed degeneration after spinal cord injury in rats and monkeys. Nat Med 3:73-76, 1997

9. Dawson TM, Dawson VL, Snyder SH: Molecular mechanisms of nitric oxide actions in the brain. Ann N Y Acad Sci 738: 76-85, 1994

10. Fehlings MG, Nguyen DH: Immunoglobulin G: A potential treatment to attenuate neuroinflammation following spinal cord injury. J Clin Immunol 30 Suppl 1: S109-112, 2010

11. Gao S, Ding J, Xiao HJ, Li ZQ, Chen Y, Zhou XS, Wang JE, Wu J, Shu WZ: Anti-inflammatory and antiapoptotic effect of combined treatment with methylprednisolone and amniotic membrane mesenchymal stem cells after spinal cord injury in rats. Neurochem Res 39: 1544-1552, 2014

12. Ha KY, Carragee E, Cheng I, Kwon SE, Kim YH: Pregabalin as a neuroprotector after spinal cord injury in rats: Biochemical analysis and effect on glial cells. J Korean Med Sci 26:404411, 2011

13. Ha KY, Kim YH, Rhyu KW, Kwon SE: Pregabalin as a neuroprotector after spinal cord injury in rats. Eur Spine $J$ 17: 864-872, 2008

14. Hulsebosch CE: Recent advances in pathophysiology and treatment of spinal cord injury. Adv Physiol Educ 26: 238-255, 2002

15. Isaksson J, Farooque M, Holtz A, Hillered L, Olsson Y: Expression of ICAM-1 and CD11b after experimental spinal cord injury in rats. J Neurotrauma 16: 165-173, 1999

16. Kaptanoglu E, Palaoglu S, Surucu HS, Hayran M, Beskonakli $\mathrm{E}$ : Ultrastructural scoring of graded acute spinal cord injury in the rat. J Neurosurg 97 Suppl 1: 49-56, 2002

17. Keane RW, Kraydieh S, Lotocki G, Bethea JR, Krajewski S, Reed JC, Dietrich WD: Apoptotic and anti-apoptotic mechanisms following spinal cord injury. J Neuropathol Exp Neurol 60: 422-429, 2001 
18. Kwon BK, Tetzlaff W, Grauer JN, Beiner J, Vaccaro AR: Pathophysiology and pharmacologic treatment of acute spinal cord injury. Spine J 4: 451-464, 2004

19. Liu XZ, Xu XM, Hu R, Du C, Zhang SX, McDonald JW, Dong HX, Wu YJ, Fan GS, Jacquin MF, Hsu CY, Choi DW: Neuronal and glial apoptosis after traumatic spinal cord injury. $J$ Neurosci 17: 5395-5406, 1997

20. Miranda KM, Espey MG, Wink DA: A rapid, simple spectrophotometric method for simultaneous detection of nitrate and nitrite. Nitric Oxide 5:62-71, 2001

21. Nakajima H, Uchida K, Yayama T, Kobayashi S, Guerrero AR, Furukawa S, Baba H: Targeted retrograde gene delivery of brain-derived neurotrophic factor suppresses apoptosis of neurons and oligodendroglia after spinal cord injury in rats. Spine 35: 497-504, 2010

22. Ohkawa H, Ohishi N, Yagi K: Assay for lipid peroxides in animal tissues by thiobarbituric acid reaction. Anal Biochem 95: 351-358, 1979

23. Oyinbo CA: Secondary injury mechanisms in traumatic spinal cord injury: A nugget of this multiply cascade. Acta Neurobiol Exp 71: 281-299, 2011

24. Park E, Velumian AA, Fehlings MG: The role of excitotoxicity in secondary mechanisms of spinal cord injury: A review with an emphasis on the implications for white matter degeneration. $J$ Neurotrauma 21: 754-774, 2004
25. Sakurai M, Nagata T, Abe K, Horinouchi T, Itoyama Y, Tabayashi $\mathrm{K}$ : Survival and death-promoting events after transient spinal cord ischemia in rabbits: Induction of Akt and caspase 3 in motor neurons. J Thorac Cardiovasc Surg 125: 370-377, 2003

26. Sullivan PG, Krishnamurthy S, Patel SP, Pandya JD, Rabchevsky AG: Temporal characterization of mitochondrial bioenergetics after spinal cord injury. J Neurotrauma 24: 991999, 2007

27. Sun Y, Oberley LW, Li Y: A simple method for clinical assay of superoxide dismutase. Clin Chem 34: 497-500, 1988

28. Tian DS, Xie MJ, Yu ZY, Zhang Q, Wang YH, Chen B, Chen $C$, Wang W: Cell cycle inhibition attenuates microglia induced inflammatory response and alleviates neuronal cell death after spinal cord injury in rats. Brain Res 1135: 177-185, 2007

29. Torres S, Salgado-Ceballos H, Torres JL, Orozco-Suarez S, Diaz-Ruiz A, Martinez A, Rivera-Cruz M, Rios C, Lara A, Collado C, Guizar-Sahagun G: Early metabolic reactivation versus antioxidant therapy after a traumatic spinal cord injury in adult rats. Neuropathology 30: 36-43, 2010

30. Vaziri ND, Lee YS, Lin CY, Lin VW, Sindhu RK: NAD(P) $\mathrm{H}$ oxidase, superoxide dismutase, catalase, glutathione peroxidase and nitric oxide synthase expression in subacute spinal cord injury. Brain Res 995: 76-83, 2004 